\title{
Ecological capability evaluation for afforestation and forest expansion using Geographic Information System (GIS) in management area of Caspian Sea
}

\author{
SEYED A. HASHEMI \\ Department of Forestry, Lahijan Branch, Islamic Azad University, Kashef Shargi street, 1616 Lahijan, Guilan, Iran
}

Manuscript received on March 15, 2018; accepted for publication on June 8, 2018

\begin{abstract}
Ecological capability evaluation is referred to as evaluation of potential capability of land and determination of its natural land use. In this paper, ecological capability of Lille Basin in Lahijan was evaluated for afforestation and forest expansion using Geographical Information Systems (GIS). For this purpose, information layers (slope, elevation, geographical directions, temperature, rainfall, humidity, soil type, and canopy cover) were prepared by digitizing the data obtained from concerned organizations. First, the layers were prepared based on coordinate systems and pixel value. Then, they were standardized using fuzzy membership functions. The evaluation criteria were prioritized with regard to expert opinion, and by using multi-criteria evaluation method (MCE) and analysis of hierarchic process (AHP), and the final weight of each criterion was determined. The weight for 8 criteria including elevation, soil type, canopy cover (\%), directions, slope, temperature, humidity, rainfall were $0.330,0.210,0.128,0.117,0.076,0.054,0.046$ and 0.210 , respectively. According to these weights, elevation has the highest value and the slope is the lowest. The results suggest that ecological capability exhibits sensitivity to variations of different criteria and its distribution is directly correlated to ecological factors especially climatic and physiographical ones. The incompatibility index (0.09) also indicated that the weights were logical. Then valuation of the maps performed. Finally, based on these values, capabilities of each environmental unit were identified and potentials of the area for afforestation were determined in five capability classes.
\end{abstract}

Key words: Ecological capability, afforestation, AHP, fuzzy, forest.

\section{INTRODUCTION}

Planning for forest regeneration and management is through decision-making processes with a hierarchic feature, which for quality of decisions on higher levels will affect those on the lower levels. These hierarchic levels can be temporal or spatial. Timing of this hierarchic structure is on three periods: long-term (strategic), middle term

E-mail: sahashemi1980@yahoo.com (tactical) and short-term (operational) (Pukkala 2002, Baskent and Keles 2005, Akinci et al. 2013). In long term planning, long term and general goals and frameworks of management objectives are determined. Long term plans are compiled for a long period. This period can be up to 50 years. In addition, like middle term, in long term planning details are not analyzed (Kangas and Kangas 2005).

Structure and concept of hierarchy can be used in a spatial scale. Spatial planning is divided 
into three levels: 1) forest mass, 2) proximity, 3) landscape (Baskent and Keles 2005). One of the most important decisions to be made in the landscape and in the long term is prioritization and siting of different functions of a forest. ECE is a useful tool for strategic planning of forests (Rossiter 1996, Siyag 2014), which predicts potential capability and the type of land use. Capabilities of a habitat are classified based on identification of its environmental and ecological characteristics. Special models are an important tool for ECE. Depending on the number of sources involved in ecological models, evaluation methods are one-criterion, two-criteria, and multi-criteria. Multi-criteria methods show land capability more coherently and more accurately as compared to one or two-criterion methods (Paulelt and Duhme 2000). The current forestry model used in Iran has seven classes (class 1 is highest quality of the vegetation habitat for timber trade). This model was used in some research activities conducted in forests in Northern Iran with the same models or in combination with criteria of other models (Adl et al. 2006). The model used in this research is general model for Iranian forests. Thus, some of its criteria should be modified for particular areas, and a special model should be prepared for each area. Modification of these criteria requires sufficient knowledge about both model and the environmental conditions.

Graymore et al. (2009) developed a framework to assess sustainability, which was used in the form a multi-criteria analysis in GIS in order to assess sustainability of sub-drainage basins. According to this paper, this framework can illustrate the needs of sub-drainage basins for reaching sustainability. The results of this combination indicated usefulness of this method for collecting a large amount of information and evaluating agriculturalecological capability ANP-DEMATEL and FAHP multi-criteria decision-making models were used to assess land suitability for agricultural land uses in outskirts of Khuzestan Province (Pourkhabbaz et al. 2015), which is not suitable for agriculture due to proximity to mountains and hills, and presence of gypsum and lime geological formations.

On the other hand, the importance of sustainable management compatible of the forest in Northern Iran is increasing. The northern forests cover approx. 1.9 million ha, of which only 1.2 million ha is taken into account as commercial forests (Zare et al. 2011). Shrinkage of these forests as the only commercial forests of Iran increases the emphasis on conserving them and the use of their wood sources. On this basis, proper management of these forests should be founded on ECE and their potentials (Store and Jokimaki 2003). Here, we discuss ecological capability evaluation for afforestation and forest expansion using GIS in Lille Basin, Gilan in order to discover advantages of GIS in forest management and ECE.

\section{MATERIALS AND METHODS}

The study area is located in Gilan Province between $50^{\circ} 0^{\prime}$ 'Eastern longitude and $37^{\circ} 11^{\prime}$ 'Northern latitude. Historically and geographically, Lahijan is a strategic region in Gilan Province, which cover an area of $407 \mathrm{~km}^{2}$ (Figure 1).

A database containing satellite images, digital topographic maps, and maps of temperature, rainfall, humidity, soil type, type and density of canopy cover and lithology (environmental factors of ecological capability evaluation) was established. When information layers were prepared and rasterized by Euclidean functions of distance or converted to raster, all of them should be standardized. This process is performed by using fuzzy membership functions. Then, Expert Choice was used to weight each information layer based on expert viewpoints in the frame of pairwise comparisons and analysis of hierarchic process (AHP) method. The data was analyzed by multi-criteria evaluation (MCE) and 


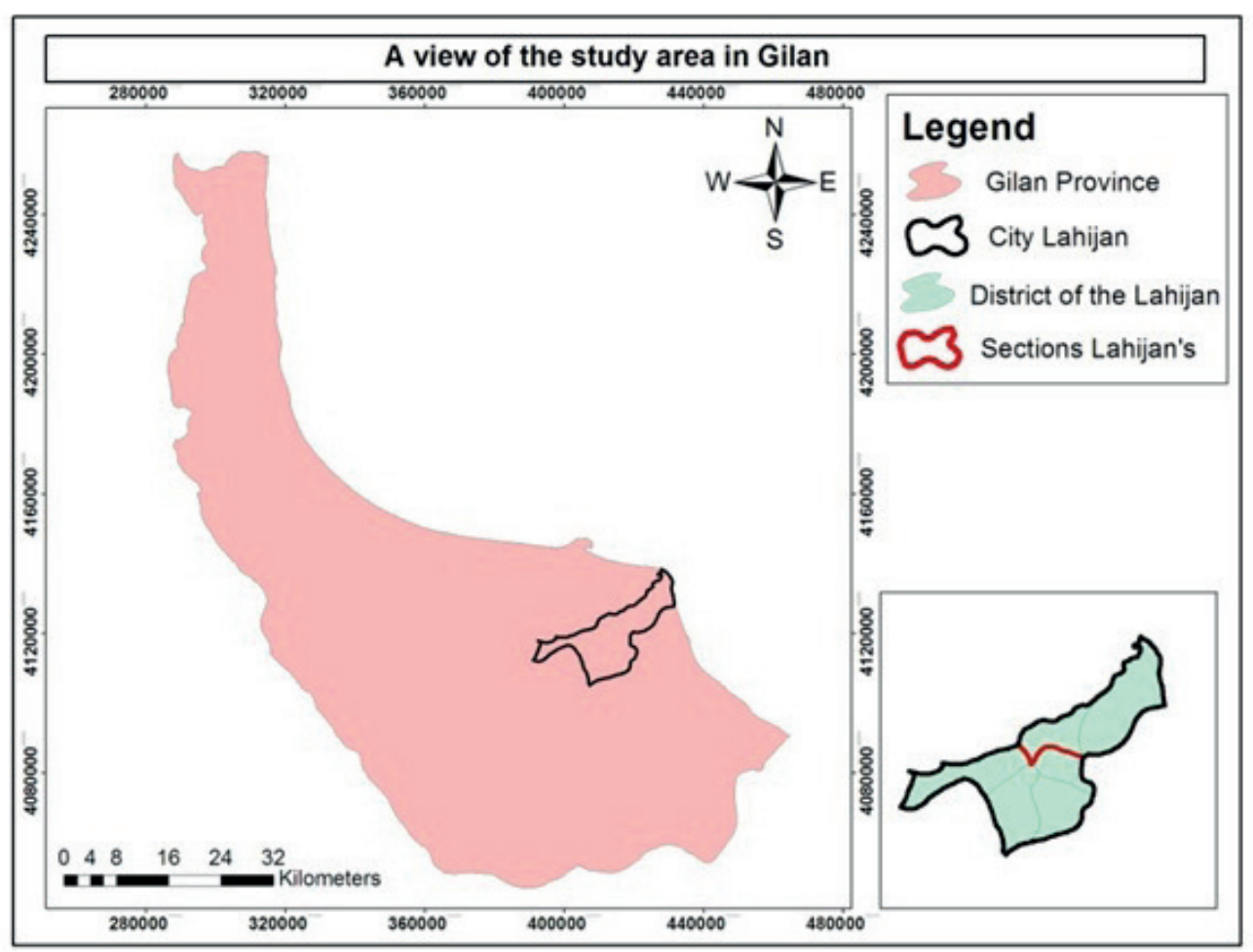

Figure 1 - Lahijan County and its districts in Gilan Province.

applied to fuzzy maps which led to an appropriate map of forest expansion potential.

Standardization or re-classification is a process in which the values of various pixels in a digital map are converted to comparable scales. Since each information layer finds a different value after processing operations. In other words, the pixel values are different before and after processing. Thus, they should be converted to comparable scales. The process of valuation of pixels of different maps and their conversion to comparable scales is known as standardization.

In this paper, fuzzy membership functions were used for standardization. In fuzzy logic, membership of an element in a set is defined within a range between 1 (full membership) and 0 (lack of full membership). Membership degree is usually expressed by a membership function, which can be in linear, non-linear, continuous, and discontinuous forms. In a fuzzy model, each pixel in a factor map is valued between 0 and 1 , which indicates suitability of the pixel location in terms of the criterion in question for the pre-determined objective. The following figure show standardized fuzzy maps (Figure 2).

\section{USE OF FUZZY OPERATORS TO COMBINE THE LAYERS WITHOUT WEIGHTING}

Fuzzy overlay functions can use joint models to combine location layers obtained from distinct sources for zonation and site selection. In this paper, first AND and Gamma fuzzy operators and then AHP pairwise comparison were used (Equations 1 and 2).

$\mu_{\text {combination }}=\operatorname{MIN}\left(\mu_{\mathrm{A}}, \mu_{\mathrm{B}}, \ldots \ldots \ldots.\right)$

AND fuzzy operator like intersection in classic sets is used when two or more criteria can help solve a problem jointly.

$\mu_{\text {combination }}=(\text { FuzzySum })^{\gamma} \times(\text { FuzzyProduct })^{1-\gamma}$

$\gamma \in[0,1]$ 

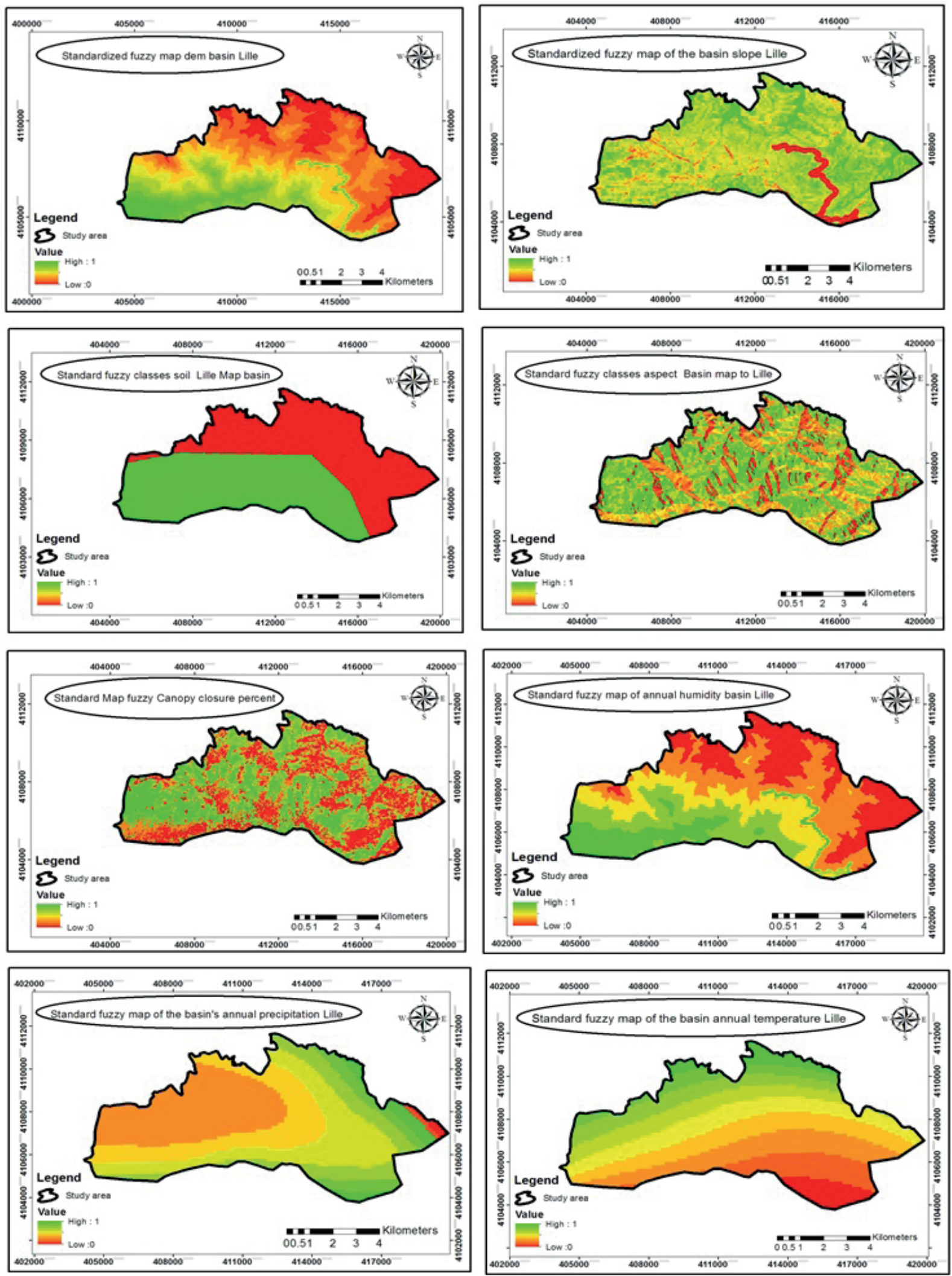

Figure 2 - Standardized fuzzy maps. 


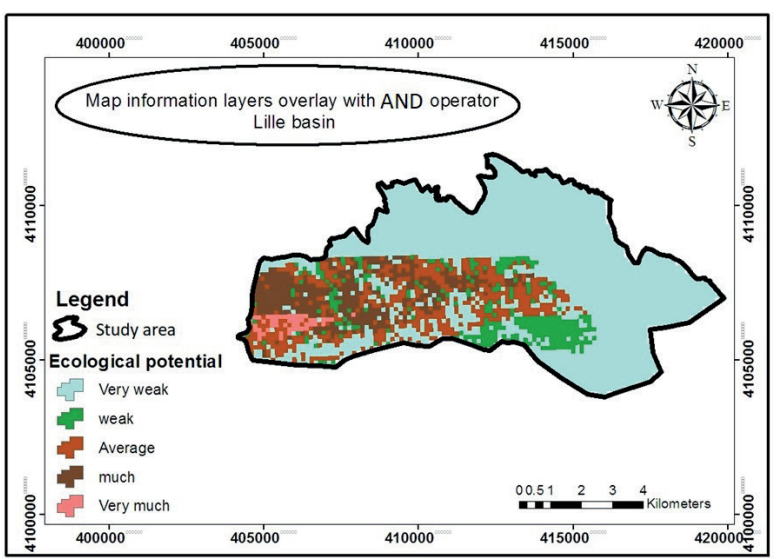

Figure 3 - Overlay map of the information layers using AND operator.

Gamma fuzzy operator is a combination of fuzzy algebraic product and fuzzy algebraic sum both raised to the power of gamma, and it is used when increasing and decreasing effects exist in interactions of the criteria (Alesheikh et al. 2008, Jiang et al. 2000).

\section{COMBINATION OF THE CRITERIA BY WEIGHTING THEM IN AHP PAIRWISE COMPARISON MATRIX}

The criteria were weighted after standardization and they were entered into AHP pairwise comparison matrix, where their weights were determined based on the expert opinions. Figure S1 (Supplementary Material) and Figure S2 show the pairwise comparison matrices, and Figure S3 show the weights resulting from comparing the criteria.

Layers of information including slope, elevation, geographical directions, temperature, rainfall, humidity, soil type and canopy cover were prepared by digitizing the data collected from concerned organizations and departments. For ecological capability evaluation, the data layers were prepared based on coordinate system and pixel value and standardized by using fuzzy membership functions. Then, the evaluation criteria were prioritized based on expert opinion, multi-criteria evaluation method, analytic hierarchic process and Expert Choice, and the final weights were given

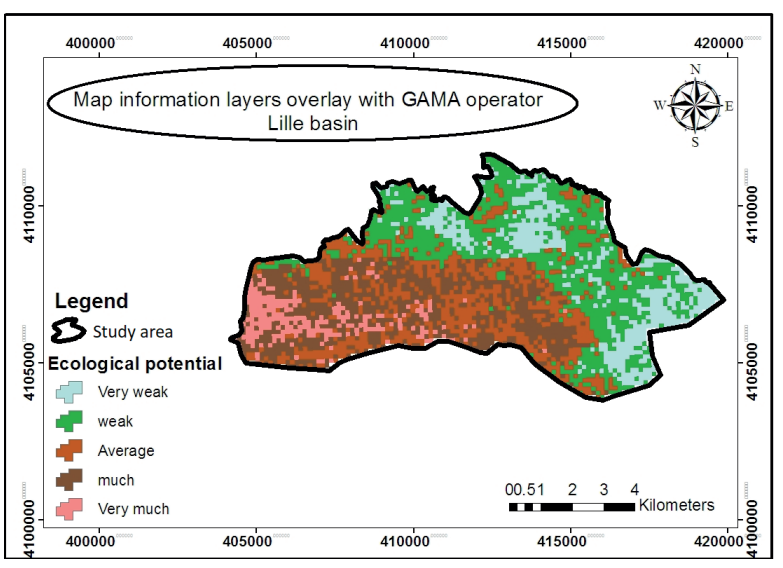

Figure 4 - Overlay map of the information layers using Gamma operator.

to the criteria. Finally, the maps were valued and relying on the existing values capability of each environmental unit and capability of the area was determined for afforestation in five classes. Since any species has specific ecological needs, adaptability of these needs to local ecological conditions is a key to successful afforestation.

\section{RESULTS}

The criteria were overlaid by using AND method and Gamma operators as well as weights obtained from pairwise comparison matrix. Figures 3, 4 and 5 show the results of this overlay. The criteria were overlaid by using AND method and Gamma operators as well as weights obtained from pairwise comparison matrix. Figures 3, 4 and 5 show the results of this overlay. The area with a very high ecological capability re-classification of overlay of the criteria using AND model is 488 hectares and the area with very high ecological capability with a gamma model of 435.9 hectares (Tables I, II, III).

Weights of eight criteria intended for this research (elevation, soil type, canopy cover, slope direction, temperature, humidity, rainfall, and slope) were $0.330,0.210,0.117,0.076,0.054$, 0.046 , and 0.210 , respectively. According to these weights, elevation has the highest and slope has the lowest values. The results suggest that ecological 


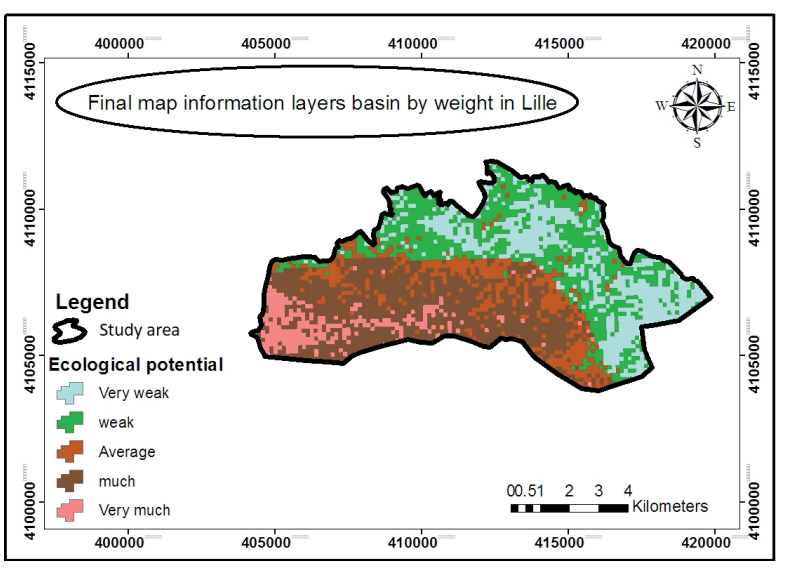

Figure 5 - Map of the re-classification of overlay of the criteria of Lille Basin using AND model.

TABLE I

Area of overlay classes using AND operator.

\begin{tabular}{cc}
\hline Ecological capability classes & Area (ha) \\
\hline very low & 4189.88 \\
low & 569.06 \\
medium & 972.23 \\
high & 729.17 \\
very high & 98.38 \\
\hline
\end{tabular}

TABLE II

Area of overlay classes using Gamma operator.

\begin{tabular}{cc}
\hline Ecological capability classes & Area (ha) \\
\hline very low & 1026.25 \\
low & 1992.70 \\
medium & 1554.80 \\
high & 15449.02 \\
very high & 435.96 \\
\hline
\end{tabular}

TABLE III

Area of overlay classes using AND operator.

\begin{tabular}{cc}
\hline Ecological capability classes & Area (ha) \\
\hline very low & 1394.69 \\
low & 1714.91 \\
medium & 912.43 \\
high & 2093.01 \\
very high & 4448.04 \\
\hline
\end{tabular}

capability is sensitive to variations of different criteria and its distribution varies with ecological factors such as climatic and physiographical factors. The inconsistency Index was $0.09(<0.1)$ which indicates that the weights above are logical and in conformity (Reshmidevi et al. 2009), and expert opinions in the frame of pairwise comparison and AHP. It also exhibits efficacy of GIS in site selection, combination and overlay of different ecological criteria.

In this paper, ecological capability of Lille Basin was evaluated with the aim of afforestation and increase in forest coverage using GIS. For this purpose, information layers including maps of slope, elevation above sea level, geographical directions, temperature, rainfall, humidity, soil type, canopy cover, were prepared by digitizing the data obtained from concerned organizations. First, data layers prepared based on coordinate systems and pixel value. Then, they were standardized using fuzzy membership functions. The evaluation criteria were prioritized with regard to expert opinion, and by using multi-criteria evaluation method (MCE), analysis of hierarchic process (AHP), and Expert Choice. Therefore, the final weight of each criterion was determined. The maps were valued and the capability of each ecological units and the entire region for afforestation was identified in five classes. Since any species has specific ecological needs, adaptability of these needs to local ecologic conditions is a key to successful afforestation. Physiographical factors including elevation variations, geographical directions, mountain hill slope has a great effect on climatic conditions of the vegetation habitat. Elevation above sea level is an indicator of climatic fluctuations. Due to elevation variations, the amount of energy received by any place on earth is different. Humidity and rainfall varies with increase in pressure and decrease in elevation. Therefore, elevation variations can influence temperature, rainfall and humidity. Pressure is low in high 
elevations. Intensity of Radiation decreases and ultraviolet rays increase. Growing period of plants becomes shorter, their pigments increase and they find a darker appearance. Relative humidity is high in the elevations. For this reason, the vegetation is mostly in the form of shrubs, which are short to withstand the winds and to survive.

The results suggested that ecological capability shows sensitivity to variations of different factors and its distribution is directly linked to ecological factors such as climatic and physiographic ones. Inconsistency index was $0.09(<0.1)$ which indicates that the weights above are logical and in conformity (Reshmidevi et al. 2009), and expert opinions in the frame of pairwise comparison and AHP. It also exhibits efficacy of GIS in site selection, combination and overlay of different ecological criteria.

\section{DISCUSSION}

Land form (slope, direction and elevation) plays an important role in evaluating the ecological capability of an area for afforestation (Kafaee 2009). When the slope of an area is not suitable, it results in limitations for afforestation operations including limited ability to work with different machineries, limited presence in the forested area, harsh climatic and environmental conditions and so on. Therefore, more gentle slopes are more desirable for afforestation. Slope was a factor of evaluation of forestry capability (Svoray et al. 2005, Ziadat and Al-bakri 2006). Northern directions on the northern hemisphere are more humid and are covered by more favorable vegetation compared to southern hemisphere (Zare et al. 2011). Also in this paper, slope direction was used as an effective factor in evaluating ecological capability of land for forestry. As elevation above sea level, absolute humidity, temperature increase growth period, and development decrease. In many other similar papers, elevation as an effective factor in capability evaluation for forestry and lower altitudes were selected as classes that are more desirable (Store and Jokimaki 2003).

Canopy cover density plays a vital role in planning for forestry (Kafaee et al. 2009). In addition, in this paper, canopy cover density obtained a high weight. For allocation of weights, due to disagreements of expert viewpoints, those of different experts were used. The highest final value was for elevation, which influences rainfall and temperature. Used multi-criteria evaluation method and GIS by eight information layers (slope, direction, hypsometry, rainfall, temperature, soil strata, geology and rivers) to introduce a favorable plant species to be planted. They included that elevation layer is more effective than other layers, which confirms the results of this research (Zare et al. 2011).

In a multi-criteria evaluation in Malaysia, the slope factor had the highest value, but in this research, it obtained the lowest weight. This shows the importance of expert opinion in a pairwise comparison, and the effect of any of these parameters is different in different places based on our research objective (Hasmadai 2009). Multi-criteria evaluation and GIS were used to find potential agricultural lands in arid and semi-arid zones in Egypt. In order to evaluate the capability of the area, all environmental parameters including physical and biological ones were analyzed and homogenous units were identified and determined (Abd El-kawy et al. 2010). In turkey, similar parameters were used to evaluate the lands for forest expansion (Dengiz et al. 2010). Therefore, by using the results of this paper, ecological capability evaluation can be implemented by means of GIS, evaluation models, and weighting models, and a plan will be prepared to facilitate planting suitable species in highly capable zones and expand their presence throughout forests in Northern Iran.

Sixty percent of the study area is covered by forests, the forested parts are of high importance 
and proper resource management within them requires planning and strong support of The Iranian Department of Environment is a governmental organization. The plans should be targeted at increase in wood production and adoption of new strategies of production, conservation and maintenance of resources with regard to ecological capability of land for afforestation. This paper can help identify potentials of forestry and its development in this region.

\section{ACKNOWLEDGMENTS}

The author appreciate Islamic Azad University Lahijan Branch making the effort to prepare this paper and Financial support by Lahijan Branch, Islamic Azad University Grant is gratefully acknowledged.

\section{REFERENCES}

ABD EL-KAWY OR, ISMAIL H, ROD J AND SULIMAN A. 2010. A Developed GIS-based Land Evaluation Model for Agricultural Land Suitability Assessments in Arid and Semi-Arid Regions. Res J Agric Biol Sci 6(5): 589-599.

ADL HR AND MARVY M. 2006. Comparative evaluation of factors of capability evaluation in three regions: Western, Eastern and Central parts of forests in Northern Iran. Ir J For Res 15(3): 289-300.

AKINCI H, OZALP A AND TURGUT B. 2013. Agricultural land use suitability analysis using GIS and AHP technique. Comput Electron Agric 97: 71-82.

ALESHEIKH A, SOLTANI M AND KHALILZADEH M. 2008. Land Assessment for Flood Spreading Site Selection Using Geospatial Information System. Int J Envi Sci Technol 5(4): 455-462.

BASKENT E AND KELES S. 2005. Spatial forest planning: a review. Ecol Model 188: 145-173.

DENGIZ O, GOL C, SARIGOLU F AND EDIS S. 2010. Parametric approach to land evaluation for forest plantation: A methodological study using GIS Model. Afr J Agric Res 5(12): 1482-1496.

GRAYMORE ML, ANNE W AND ANNEKE R. 2009. An index of regional sustainability: a GIS-based multiple criteria analysis decision support system for progressing sustainability. Ecol Complex 6(4): 453-462.

HASMADAI I. 2009. Developing policy for suitable harvest zone using multi criteria evaluation and GIS-based decision support system. Int J Econ Finan 1(2): 105-117.
JIANG H AND EASTMAN R. 2000. Application of fuzzy measurement in multi-criteria evaluation in GIS. Int $\mathrm{J}$ Geogr Inf Sci 14(2): 173-184.

KAFAEE BS. 2009. Environmental evaluation of forests for land classification using GIS case study: Kazemrud, Basin-Northern Forests of Iran. Ol Kesh Journal 12(1): 67-80.

KANGAS J AND KANGAS A. 2005. Multiple criteria decision support in forest management- the approach, methods applied, and experiences gained. Forest Ecol Manag, p. 133-143.

PAULELT S AND DUHME F. 2000. GIS Assessment of Munich's urban forest structure for urban planning. Arboric J 26(3): 133-141.

POURKHABBAZ H, AGHDAR H, MOHAMMADYARI F AND JAVANMARDI S. 2015. Land suitability evaluation for agricultural land uses by multi-criteria decision-making models. J Environ Sci 41: 429-445.

PUKKALA T. 2002. Multi-objective Forest Planning. Kluwer Academic publisher, Dordrecht, Netherlands, 200 p.

RESHMIDEVI TV, ELDHO T AND JANA R. 2009. A GISintegrated fuzzy rule-based inference system for land suitability evaluation in agricultural watersheds. Agric Syst 101: 101-109.

ROSSITER DG. 1996. A rhetorical framework for land evaluation. Geoderama 72: 165-190.

SIYAG PR. 2014. Afforestation, reforestation and forest restoration in arid and semi-arid tropics, a manual of technology and management. Springer, $300 \mathrm{p}$.

STORE R AND JOKIMAKI J. 2003. A GIS-based multiscale approach to habitat suitability modeling. Ecol Model 169: $1-15$.

SVORAY T, BAR P AND BANNET T. 2005. Urban land use allocation in a Mediterranean ecotone: habitat heterogeneity model incorporated in a GIS using a multicriteria mechanism. Landsc Urban Plan 72: 337-351.

ZARE R, BABAEIKAFAKI S AND MATTAJI A. 2011. Suggestion the Appropriate Species for afforestation in South Hillside of Alborz Mountain by Using GIS (Case Study: Dareh Vesieh Basin). J Res Renew Nat Resou 2(1): 55-67.

ZIADAT FM AND AL-BAKRI JT. 2006. Comparing existing and potential use for sustainable land utilization. Jordan $\mathrm{J}$ Agric Sci 4: 327-386.

\section{SUPPLEMENTARY MATERIAL}

Figure S1 - Pairwise comparison matrix.

Figure S2 - Pairwise comparison matrix.

Figure S3 - The resulting weights of the criteria. 